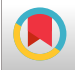

\title{
Prevalence of Kidney, Renal Pelvis, and Ureter Cancers in Isfahan Province, Iran
}

\author{
Zahra Tolou-Ghamari ${ }^{1, *}$ \\ ${ }^{1}$ Isfahan Kidney Transplantation Research Center, Department of Urology, Alzahra Research Institute, Isfahan University of Medical Sciences, Isfahan, Iran \\ "Corresponding author: Isfahan Kidney Transplantation Research Center, Department of Urology, Alzahra Research Institute, Isfahan University of Medical Sciences, Isfahan, \\ Iran.Email: toloeghamari@pharm.mui.ac.ir
}

Received 2019 June 17; Revised 2019 July 01; Accepted 2019 July 01.

\begin{abstract}
Background: According to the literature, the incidence of kidney cancer is on the rise worldwide.

Objectives: The aim of this investigation was to provide the data of period prevalence of kidney, renal pelvis, and ureter cancers in the general population including the military or non-military population in Isfahan province, Iran.

Methods: Data associated with cancers of the kidney, renal pelvis, and ureter were obtained from the Isfahan Cancer Registry between 2011 and 2015. Kidney, renal pelvis, and ureter cancers were defined by topography codes of C64, C65, and C66, respectively. Results: There were 404, 21, and 18 registered cases of kidney, renal pelvis, and ureter cancers, respectively, corresponding to a PP of 8.9 per 100000 population. The mean \pm SD of age was $56.6 \pm 16.8$ years in patients. The age of kidney cancer patients was more than 50 years in 51\% of the cases. The incidences were 2.0 in 2011 - 2012, 2.2 in 2012 - 2013, 2.3 in 2013 - 2014, and 2.4 in 2014 - 2015 per 100000 population. The death data confirmed $14 \%$ and $86 \%$ mortality associated with kidney cancer and renal pelvis, respectively. Conclusions: The incidence rate of kidney cancer showed increases by $20 \%$. The data confirmed high mortality associated with cancer of renal pelvis. In order to decrease cancer incidence, healthcare planning should be based on military management together with a well-structured pharmacotherapy strategy.
\end{abstract}

Keywords: Kidney, Cancer, Prevalence, Renal Pelvis

\section{Background}

Accounting for $2 \%$ of adult malignancies, renal cell carcinoma (RCC) takes third place among urologic tumors with multiple clinical aspects associated with its expansion. Differences in age, gender, and geographic distribution have been detailed. The prevalence of RCC has risen steadily and it has the highest mortality (around $40 \%$ ) among the genitourinary cancers. Its maximum incidence was reported in the sixth decade of life with more frequency in Afro-American people (1-3). Among men and women, this malignancy takes the tenth and fourteenth places, respectively (4). RCC characterizes $85 \%$ to $90 \%$ of renal malignancies $(5,6)$. There is a strong correlation between smoking and RCC (7). Kidney cancer comprises malignant tumors arising from the parenchyma and renal pelvis in adults. Regarding the incidence rate of RCC, there is variability in worldwide reports with a high rate reported in Europe and North America and a low rate in Asia and South America (8). The highest incidence rate was found in Holland with values of 16.9 and 9.2 per 100000 population in males and females, respectively. The lowest incidence was 8.2 in males and 3.7 in females per 100000 population in Spain (9-12). Falebita et al. associated these findings with better diagnosis and registration (13). As the third province in terms of population (4982100 people) after Tehran and Mashhad cities, we tried to define the period prevalence and incidence rate in Isfahan province, Iran.

\section{Objectives}

This study was designed to provide the most efficient data associated with the incidence of kidney, renal pelvis, and ureter cancers, as well as the mortality pattern in both military and non-military families.

\section{Methods}

Kidney, renal pelvis, and ureter cancers data were obtained from the Isfahan Cancer Registry, located at the Isfahan Deputy of Health (2011 - 2015). The study was conducted at the Isfahan Kidney Transplantation Research Center (IKTRC) and its protocol was reviewed for ethical 
purposes. Ethical clearance was issued by the Deputy of Research. The paper was extracted from a project with a certificate number of 295115.

The Isfahan Cancer Program is to record all cancer cases in military or non-military families in Isfahan. All pathology centers and other information resources such as hospitals, provincial mortality databases, hematologyoncology centers, radiotherapy centers, etc., are obliged to report their data to the Isfahan Cancer Office of Disease Control and Prevention (CDC). The management arm of the program is the Deputy of Research at the Isfahan University of Medical Sciences. The cancer types under study were defined according to the International Classification of Diseases (ICD-O; third edition). Kidney, renal pelvis, and ureter cancers were defined by the topography codes, C64, C65, and C66, respectively. Data, with linkage to using of the de-identified patient name and surname, demographic data such as age and gender, the final code for cancer diagnosis, and date of cancer report were recorded. The total population of Isfahan city was obtained from the Isfahan Program and Budget Management Organization. The period prevalence (PP) was calculated as the proportion of total cases over the period of 2011 - 2015 divided by the population at risk during the same period multiplied by 100000 . The incidence rate (IR) was calculated by dividing new cases of cancer during a given period to the population at risk during the same period multiplied by 100000. The statistical analysis of d-Base was performed using Microsoft Excel and SPSS V. 20 (Chicago, IL, USA) for Windows (14-20).

\section{Results}

The demographic and epidemiologic characteristics of patients with kidney cancer are shown in Table 1. For a period of four years, there were 404 (145 females and 258 males), 21 (8 females and 13 males), and 18 ( 2 females and 16 males) recorded cases of kidney, renal pelvis, and ureter cancers, respectively. The Kolmogorov - Smirnov test was used to examine the normal distribution of age. The mean age (SD, range) was 56.6 years $(16.8 ; 2$ - 93) for kidney cancer, 68.5 years $(13.8 ; 46$ - 91) for renal pelvis cancer, and 65 years (11.1; 44 - 83) for ureter cancer. As shown in Figure 1, the most incidence of kidney cancer (51\%) was between the ages of 50 to 70 years old. Figure 2 shows the age distribution in patients with renal pelvis and ureter cancers. The calculated PP was 8.1, 0.42, and 0.4 per 100000 population for kidney, renal pelvis, and ureter cancers, respectively. There were 56 and 18 deaths associated with kidney and renal pelvis cancers, respectively, while no death was recorded for ureter cancer. As shown in Figure 3, the IRs were 2, 2.2, 2.3, and 2.4

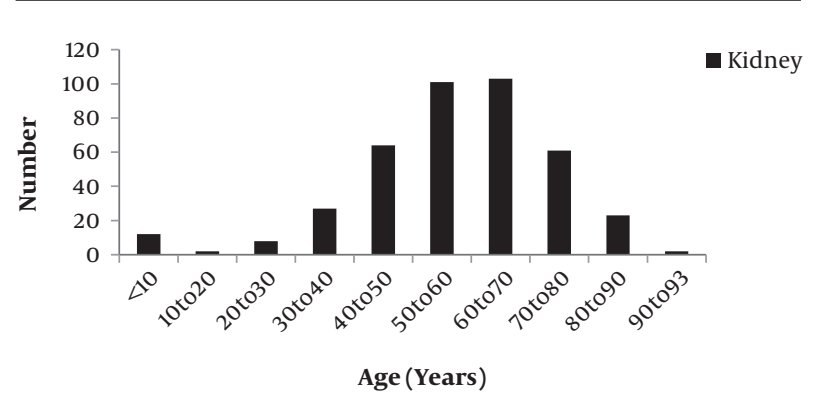

Figure 1. Distribution of age in patients with kidney cancer

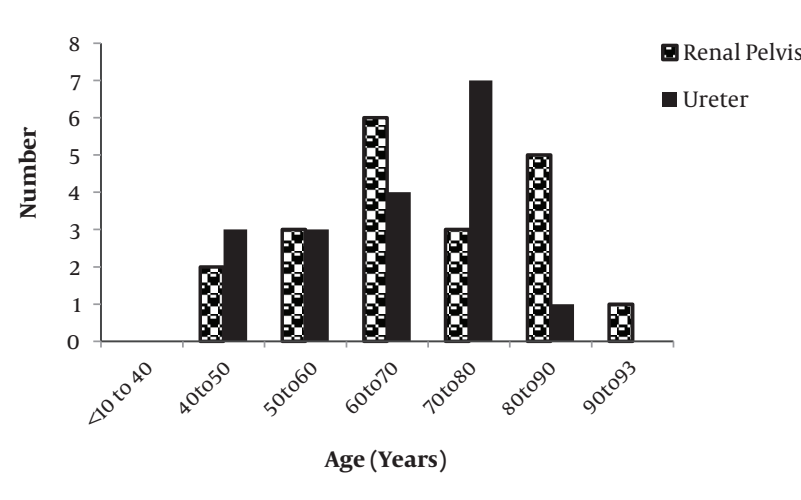

Figure 2. Distribution of age in patients with renal pelvis and ureter cancers

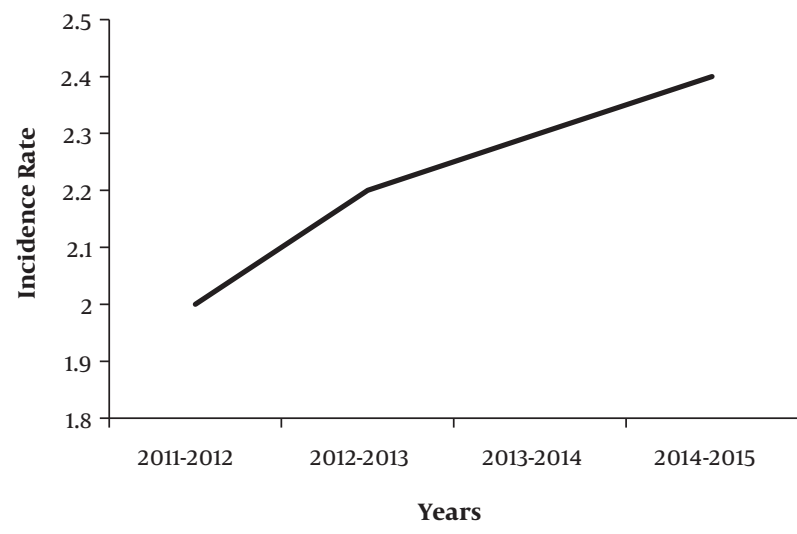

Figure 3. Incidence rate of kidney cancer associated with gender

for the years 2011 - 2012, 2012 - 2013, 2013 - 2014, and 2014 2015 , respectively.

\section{Discussion}

This study presented the most updated profile of incidence and mortality associated with kidney, renal pelvis and ureter cancers and trends based on high-quality data. 


\begin{tabular}{lcccccc}
\hline Table 1. Demographic Characteristics in the Study Population & No. & Min & Max & Mean & SD & P Value \\
\hline Patients with kidney cancer (group 1) & 403 & 2 & 93 & 56.5 & 16.7 & $0.799(1$ vs. 2); \\
Patients with renal pelvis cancer (group 2) & 20 & 46 & 91 & $0.426(1$ vs. 3); \\
Patients with ureter cancer (group 3) & 18 & 44 & 83 & 65 & 11 \\
\hline
\end{tabular}

Kidney cancer among adults comprises malignant tumors arising from the renal parenchyma and renal pelvis. Renal parenchyma cancer is the predominant kidney cancer, mainly of the adenocarcinoma cell type (renal cell cancer). The highest incidence rates were observed in Eastern Europe and North America while its mortality rates were the highest in European countries with an estimated economic burden of 1.6 billion dollars (21). Renal pelvis cancers are mostly of the transitional cell type (8). Renal cell carcinoma (RCC) is the third urologic tumor in incidence and has the highest mortality rate of the genitourinary cancers.

A recent study evaluated the incidence rate of kidney cancer in Iran. The results confirmed that the incidence rate of kidney cancer is low in Iranian men (ASR $=1.94$ per 100000 population). Around $2 \%$ of adulthood and $2 \%$ $6 \%$ of childhood renal malignancies are RCC. In agreement with a previous study (22), the male PP (=11.3) was $80.9 \%$ higher than the female $\mathrm{PP}(=6.4)$ in the current study.

Previous reports confirm that the increasing incidence in recent decades is associated with a better diagnosis of the disease. In the current study, there was a $20 \%$ increase in incidence from 2011 to 2015 . The prevalence rates of renal pelvis and ureter cancers were 0.42 and 0.4 , respectively, confirming that transitional cell carcinomas of the ureter and renal pelvis are relatively uncommon (3, 21-24).

According to the GLOBOCAN statistics (2012), there were more than 1000 new cases and 400 more deaths in Canada. The number of RCC by the year 2020 is estimated at 4139 (with 1373 deaths) in males versus 2625 (with 972 deaths) in females (25).

In this study, there were the death rates of $14 \%, 86 \%$, and $0 \%$ associated with kidney, renal pelvis, and ureter cancers, respectively. The previous publication reported that several factors could affect the incidence and mortality of cancer in different racial and geographical parts of the world, including the rate of screening performance in order to manage the risk of kidney cancer, accidental diagnosis, genetic and environmental risk factors, and socioeconomic status. Prominent risk factors comprise cigarette smoking, obesity, and hypertension. Accumulating evidence suggests an etiologic role for physical inactivity, alcohol consumption, high parity among women, and occupational exposure to trichloroethylene. Modifications in lifestyle could change many of the mentioned risk factors, suggesting a strong prospect for intervention $(3,21-26)$.

\subsection{Conclusions}

The incidence rate of kidney cancer showed increases by $20 \%$ in this study. Reported data confirmed high mortality associated with renal pelvis cancer. Therefore, we propose approaches to increase service availability for patients with signs of cancer to facilitate appropriate diagnosis. In addition, studies that concern population-based designs, such as representative surveys that collect data directly from individuals, could supplement data for more precise estimation of cancer incidence and mortality.

This study was based on available recorded data associated with kidney cancer from military and non-military people. The results of this study cannot be extended to individualized pharmacotherapy such as previously published reports in the Iranian population (27-30). Kidney cancer is a condition that needs both pharmacotherapy and surgical management. Taking this study as a reference, further research involving updated sample sizes is proposed to plan. Finally, in order to provide satisfying guidelines, this investigation could be used for supportive care that extends to both military and non-military population.

\section{Acknowledgments}

The author appreciates the contribution of the staff of Cancer Registry located at the Deputy of Health and expresses sincere thanks to Professors Hamid Mazdak (head of department), Mehrdad Mohammadi (head of Isfahan Kidney Transplantation Research Center), and Massieh Sabouri (head of Alzahra Research Center).

\section{Footnotes}

Conflict of Interests: The author declares no conflict of interest in this study.

Ethical Considerations: This study protocol has been reviewed for ethical purposes. Ethical clearance was issued by Deputy of Research. This study is extracted from a project with certificate number of 295115. 
Financial Disclosure: There was not any financial disclosure directly for this study.

Funding/Support: There was not any funding associated to this study.

\section{References}

1. John J, Aldera AP, Sinha S, Lazarus J. Clear cell renal cell carcinoma with a ureteral thrombus. J Endourol Case Rep. 2018;4(1):159-62. doi: 10.1089/cren.2018.0067. [PubMed: 30426074]. [PubMed Central: PMC6225074].

2. Cairns P. Renal cell carcinoma. Cancer Biomark. 2010;9(1-6):461-73. doi: 10.3233/CBM-2011-0176. [PubMed: 22112490]. [PubMed Central: PMC3308682].

3. Pascual D, Borque A. Epidemiology of kidney cancer. Adv Urol. 2008:782381. doi: 10.1155/2008/782381. [PubMed: 19009036]. [PubMed Central: PMC2581742].

4. Algaba F, Moreno A, Trias I. Tumores renales. Uropatologia tumoral. Barcelona, Spain: Pulso Ediciones; 1996. p. 21-101. Spanish.

5. Robles JE, Rosell D, Zudaire JJ, Berian JM. [Epidemiology of renal parenchymal tumors].J Med Univ Navarra. 1999;43(2):68-76. Spanish.

6. Jemal A, Siegel R, Ward E, Murray T, Xu J, Smigal C, et al. Cancer statistics, 2006. CA Cancer J Clin. 2006;56(2):106-30. [PubMed: 16514137].

7. Vogelzang NJ, Stadler WM. Kidney cancer. Lancet. 1998;352(9141):16916. doi:10.1016/S0140-6736(98)01041-1. [PubMed: 9853456].

8. Chow WH, Dong LM, Devesa SS. Epidemiology and risk factors for kidney cancer. Nat Rev Urol. 2010;7(5):245-57. doi:10.1038/nrurol.2010.46. [PubMed: 20448658]. [PubMed Central: PMC3012455].

9. Stang A, Buchel C. A novel approach for estimating the nationwide incidence of renal cancer. Emerg Themes Epidemiol. 2014;11:8. doi: 10.1186/1742-7622-11-8. [PubMed: 25057278]. [PubMed Central: PMC4108273]

10. van de Schans SA, Aben KK, Mulders PF, Haanen JB, van Herpen C, Verhoeven $\mathrm{RH}$, et al. Modest improvement in 20 years of kidney cancer care in the Netherlands. Eur J Cancer. 2012;48(12):1822-30. doi: 10.1016/j.ejca.2012.01.033. [PubMed: 22365640].

11. Cleries R, Esteban L, Borras J, Marcos-Gragera R, Freitas A, Carulla M, et al. Time trends of cancer incidence and mortality in Catalonia during 1993-2007. Clin Transl Oncol. 2014;16(1):18-28. doi: 10.1007/s12094-0131060-y. [PubMed: 23740137].

12. Souza DL, Bernal MM. [Incidence, prevalence and mortality of kidney cancer in Spain: Estimates and projections for the 1998-2022 period]. Actas Urol Esp. 2012;36(9):521-6. Spanish. doi: 10.1016/j.acuro.2011.09.003. [PubMed: 22196967].

13. Falebita OA, Mancini S, Kiely E, Comber H. Rising incidence of renal cell carcinoma in Ireland. Int Urol Nephrol. 2009;41(1):7-12. doi: 10.1007/s11255-008-9413-0. [PubMed: 18566909].

14. Mazdak H, Tolou-Ghamari Z. Preliminary study of prevalence for bladder cancer in Isfahan Province, Iran. Arab J Urol. 2018;16(2):206-10. doi: 10.1016/j.aju.2017.11.017. [PubMed: 29892483]. [PubMed Central: PMC5992262].

15. Tolou Ghamari Z. Prevalence of lung cancer in Isfahan province, Iran. J Egypt Natl Canc Inst. 2018;30(2):57-9. doi: 10.1016/j.jnci.2018.03.001. [PubMed: 29691096].

16. Tolou-Ghamari Z, Tadayon F, Mazdak H. Prevalence of liver cancer in Isfahan province, Iran. Indonesia J Cancer. 2018;12(2):56. doi 10.33371/ijoc.v12i2.578.

17. Tolou-Ghamari Z. Prevalence of breast cancer in Isfahan province, Iran. Women Health Bull. 2018;5(4). doi: 10.5812/whb.82678.

18. Tolou-Ghamari Z. Thyroid cancer in Isfahan province, Iran; prevalence and demographic characteristics. Jundishapur J Chron Dis Care. 2019;8(1). doi:10.5812/jjcdc.86864.

19. Tolou-Ghamari Z. Prevalence of stomach cancer in Isfahan province, Iran. GulfJ Oncol. 2018;8(1). doi: 10.5812/whb.82678.

20. Tolou-Ghamari Z. Prevalence of skin cancer in Isfahan province, Iran Jentashapir J Health Res. 2018;9(2). doi: 10.5812/jjhr.82743.

21. Wong MCS, Goggins WB, Yip BHK, Fung FDH, Leung C, Fang Y, et al. Incidence and mortality of kidney cancer: Temporal patterns and global trends in 39 countries. Sci Rep. 2017;7(1):15698. doi: 10.1038/s41598-01715922-4. [PubMed: 29146923]. [PubMed Central: PMC5691143].

22. Hassanipour S, Namvar G, Fathalipour M, Salehiniya H. The incidence of kidney cancer in Iran: A systematic review and meta-analysis. Biomedicine (Taipei). 2018;8(2):9. doi: 10.1051/bmdcn/2018080209. [PubMed: 29806587]. [PubMed Central: PMC5992926].

23. Medina-Rico M, Ramos HL, Lobo M, Romo J, Prada JG. Epidemiology of renal cancer in developing countries: Review of the literature. Can Urol Assoc J. 2018;12(3):E154-62. doi: 10.5489/cuaj.4464. [PubMed 29283089]. [PubMed Central: PMC5869042].

24. Kirkali Z, Tuzel E. Transitional cell carcinoma of the ureter and renal pelvis. Crit Rev Oncol Hematol.2003;47(2):155-69. [PubMed:12900009].

25. GLOBOCAN 2012. Cancer incidence, mortality and prevalence worldwide. Canada: Kidney; 2017.

26. Mohammadian M, Pakzad R, Towhidi F, Makhsosi BR, Ahmadi A, Salehiniya $\mathrm{H}$. Incidence and mortality of kidney cancer and its relationship with HDI (Human Development Index) in the world in 2012. Clujul Med. 2017;90(3):286-93. doi: 10.15386/cjmed-691. [PubMed: 28781525]. [PubMed Central: PMC5536208].

27. Tolou-Ghamari Z, Palizban AA. Laboratory monitoring of cyclosporine pre-dose concentration (C0) after kidney transplantation in Isfahan. Iran J Med Sci. 2015;28(2):81-5.

28. Tolou-Ghamari Z, Shaygannejad V, Ashtari F. Preliminary study related the incidence of methylprednisolone pulse therapy in patients visited multiple sclerosis clinic located at the Isfahan Kashani Hospital. Int J Prev Med. 2013;4(Suppl 2):S274-8. [PubMed: 23776737] [PubMed Central: PMC3678231].

29. Shaygannejad V, Tolou-Ghamari Z. What is the real fate of vitamin D in multiple sclerosis? Int JPrev Med.2013;4(Suppl 2):S159-64. [PubMed: 23776718]. [PubMed Central: PMC3678212].

30. Tolou-Ghamari Z, Mehavari Habibabadi J, Palizban AA. Evidencebased pharmacotherapy of epilepsy. Arch Neurosci. 2014;2(2). doi: 10.5812/archneurosci.18468. 